\title{
Modeling the outflow of liquid with initial supercritical parameters using the relaxation model for condensation
}

\author{
Sergey Lezhnin ${ }^{1,2}$, Maksim Alekseev ${ }^{1,{ }^{*}, \text { Ivan }_{\text {Vozhakov }}{ }^{1,2} \text {, and Nikolay Pribaturin }}{ }^{1}$ \\ ${ }^{1}$ Kutateladze Institute of Thermophysics SB RAS, Novosibirsk, Russia \\ ${ }^{2}$ Novosibirsk State University, Novosibirsk, Russia
}

\begin{abstract}
The two-temperature model of the outflow from a vessel with initial supercritical parameters of medium has been realized. The model uses thermodynamic non-equilibrium relaxation approach to describe phase transitions. Based on a new asymptotic model for computing the relaxation time, the outflow of water with supercritical initial pressure and super- and subcritical temperatures has been calculated.
\end{abstract}

\section{Introduction}

Depressurization of vessels and pipelines in power plants, filled with water under high pressure, results in superheated water outflow.

Such a sharp decrease in pressure leads to a generation of compression waves in the area around the separation zone and further formation of the jet of the boiling coolant [1]. If in the stationary mode both pressure and temperature are supercritical $\left(p>p_{C R}, T>T_{C R}\right)$, it is possible for fluid parameters to approach the binodal (boundary of "single-phase and two - phase" region) from the condensation curve [2]. The process of adiabatic expansion, as a rule, is non-equilibrium, and thermodynamic non-equilibrium two-temperature model of steam-water mixture motion is frequently used for its description [3]. Simulating vaporization in real conditions requires either setting the parameters of the heterogeneous mechanism of bubbles formation (e.g. [4]), or using a different (phenomenological) approach (e.g., relaxation one [5]). In particular, in [4] it is proposed to specify the number of heterogeneous nucleation sites as an empirical parameter. In addition, asymptotic models of bubble growth are applied. Due to the use of bubble structure alone to describe the twophase medium, this approach loses its adequacy at large $(\alpha>0.3-0.5)$ volumetric void fractions. Earlier in [6] the authors, based on the performed analysis, proposed a simple relaxation model to describe thermal and dynamic processes, accompanied by water boiling-up at depressurization of high-pressure vessel. The model implies the droplet structure of the liquid-vapor flow, which is typical for high differential pressure between the vessel and atmosphere. In this paper we generalize this model for the case of nonequilibrium condensation.

\footnotetext{
* Corresponding author: almaxcom@mail.ru
} 


\section{Governing equations}

Numerical simulation is carried out in the no-slip phase condition and given the fact that the vapor and liquid phases can be in a metastable state. The system of the model equations includes the Euler equations in the axisymmetric approximation:

$$
\begin{aligned}
& \frac{\partial}{\partial t}(\rho r)+\frac{\partial}{\partial z}(\rho r u)+\frac{\partial}{\partial r}(\rho r v)=0 \\
& \frac{\partial}{\partial t}(\rho r u)+\frac{\partial}{\partial z}\left(\rho r u^{2}\right)+\frac{\partial}{\partial r}(\rho r u v)=-r \frac{\partial p}{\partial z} \\
& \frac{\partial}{\partial t}(\rho r v)+\frac{\partial}{\partial z}(\rho r u v)+\frac{\partial}{\partial r}\left(\rho r v^{2}\right)=-r \frac{\partial p}{\partial r} \\
& \frac{\partial}{\partial t}(E r)+\frac{\partial}{\partial z}(u r(E+p))+\frac{\partial}{\partial r}(v r(E+p))=0
\end{aligned}
$$

added with the equation for interphase mass transfer:

$$
\begin{gathered}
\frac{\partial(\rho r X)}{\partial t}+\frac{\partial(\rho r u X)}{\partial z}+\frac{\partial(\rho r v X)}{\partial r}=\Gamma \\
\Gamma=\frac{\rho\left(X_{S}-X\right)}{\theta}
\end{gathered}
$$

Here, $z, r$ are the axial and radial coordinates, $u, v$ are the axial and radial velocity components, $\rho$ is the density, $p$ is the pressure, $E$ is the specific internal energy, $X$ is the steam quality, and $X_{S}$ is the equilibrium steam quality (at given $p$ and $E$ ), $\Gamma$ is the vapor generation rate, and $\theta$ is the relaxation time. The system of equations (1)-(6) is closed by the equation of state (relationship between $p, E, \rho, X)$.

\section{Phase transition relaxation model}

The work [6] proposed the analytical relaxation model, based on the analysis of interfacial heat fluxes "steam - superheated liquid". The model, designed only to study the nonequilibrium boiling, is based on calculation of the nonstationary temperature field in the superheated water drops. Due to low vapor density the droplet structure of the two-phase medium is characteristic even at small steam quality (but moderate and large volumetric void fractions) [7]; and it may be used in the modeling of intensive evaporation of liquid. The average droplet size is determined from the simplified analysis of instability of the "water-steam" interface; and the size of droplets corresponds to the wave length, the fastest in destroying the interfacial surface, and is determined by a critical Weber number $W e_{C R} \approx 2 \pi$ (water, liquid metals) [7,8]. According to the model, at the micro-level of drops, heat required for vaporization is supplied from the superheated liquid. The temperature of the droplet surface is equal to steam temperature [9].

To calculate the relaxation time the following formula is proposed [6]: 


$$
\theta=\left\{\begin{array}{l}
\frac{\sigma^{2} \rho_{L}^{2}}{4 a_{L} \rho_{V}^{2}\left(P-P_{S}\right)^{2}}, P-P_{S}>\frac{\rho_{L} L^{2}}{2\left(\frac{\rho_{L}}{\rho_{V}}-1\right)^{2} C_{P, L} T} \\
\frac{\sigma^{2}\left(\frac{\rho_{L}}{\rho_{V}}-1\right)^{4} C_{P, L}^{2} T^{2}}{a_{L} \rho_{V}^{2} L^{4}}, P-P_{S} \leq \frac{\rho_{L} L^{2}}{2\left(\frac{\rho_{L}}{\rho_{V}}-1\right)^{2} C_{P, L} T}
\end{array}\right.
$$

Here, $\sigma$ is the surface tension coefficient, $a$ is the thermal diffusivity, and $L$ is the enthalpy of phase transition.

For the relaxation model to be used not only at evaporation, but condensation as well, it was necessary to substantially modify the equation of two-phase medium state, which closes the system of equations (1)-(6) in case of the existence of not only saturated, but super-cooled steam.

Although the size of drops formed in the condensation may differ from the case of the jet breakup [7,8], the test calculations were based on the formula (7).

\section{Modeling results and discussion}

In the work, the calculations of water outflow at initial supercritical parameters conducted according to the equilibrium and non-equilibrium relaxation model of phase transition were compared. Thermodynamic equilibrium model of two-phase medium was, in fact, obtained from the full system (1)-(4) with its closure by the equation of state of the equilibrium twophase mixture A more precise analysis showed that in the considered range of the calculated parameters, the equilibrium model was adequate with sufficient accuracy at $\theta<10^{-5}$ s.

The value $\theta$ is proportional to the square of the droplet size, i.e. is very sensitive to the value of this parameter. The size of the drop itself, according to the model, is determined by the value of Weber number $\mathrm{We}_{\mathrm{CR}}$ and, formally, has to reach this value asymptotically at large times. At initial design parameters the formula (7) gives clearly lower value $\theta<10^{-8} \mathrm{~s}$ (corresponding droplet radius $R_{D R O P}<10^{-7} \mathrm{~m}$ ). Therefore, in numerical modeling, higher fixed relaxation times were taken.

Figure 1 shows calculation results for axial profiles of steam quality for the case of depressurization at the end of pipe with water coolant in the model liquid-vapor atmosphere [6]. Profiles are calculated at time $60 \mu \mathrm{s}$ after depressurization at different values of relaxation time $\theta$.

As noted above, when $\theta=10^{-5} \mathrm{~s}$ the results of calculation based on the relaxation approach do not significantly differ from those of the equilibrium thermodynamic model. As it was shown earlier [2] a small variation of initial parameters of the supercritical region can lead to a change in direction (sign) of phase transformation. Figure 1a) shows the process of coolant vaporization, while Fig. 1b) presents the process of condensation.

In numerical computation, the "local" value of relaxation time $\theta$ was determined for control, and parameters (density, temperature...) included in the expression (7) were considered as variables. It turned out that both in the case of evaporation and condensation in these calculations, the relaxation time ranges from $10^{-6}$ to $10^{-3} \mathrm{~s}$, and in the main array, from $10^{-5}$ to $10^{-4} \mathrm{~s}$. These values are in good agreement with experimental data on relaxation time at steam condensation in Laval nozzles. In particular, in [10] it was shown that the characteristic relaxation time is of the order of $10^{-5} \mathrm{~s}$. 
The proposed model needs further refinement. Its main disadvantage is "rough estimation" of the most important parameter, the radius of the droplet. Apparently, the drop radius is an empirical parameter that must be determined from the analysis of appropriate experimental data. There is also a need in a more reasoned approach to the choice of drop sizes to describe the condensation process.

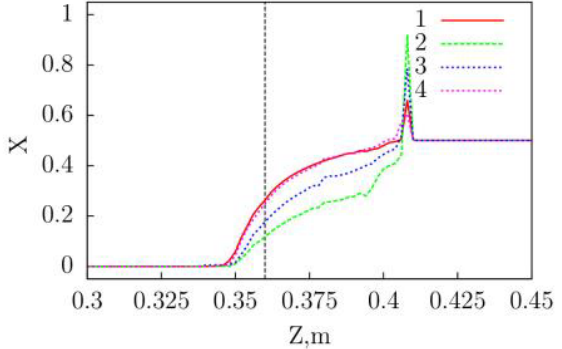

a

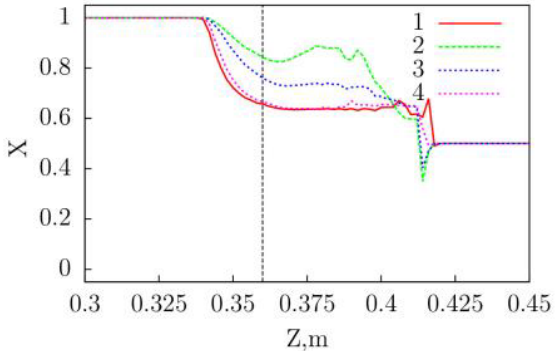

b

Fig. 1. Profiles of steam quality at time $\mathrm{t}=60 \mu$ s for different values of relaxation time. a) $\mathrm{P}=23 \mathrm{MPa}$, $\mathrm{T}=643 \mathrm{~K}, \mathrm{~b}) \mathrm{P}=23 \mathrm{MPa}, \mathrm{T}=653 \mathrm{~K}(1-$ equilibrium model of phase transition, $2-\theta=10-4 \mathrm{~s}, 3-$ $\theta=5 \cdot 10-5 \mathrm{~s}, 4-\theta=10-5 \mathrm{~s})$, the vertical line is the coordinate of the channel end.

\section{Conclusion}

The non-equilibrium relaxation model describing the processes of superheated coolant boiling-up has been generalized to describe non-equilibrium condensation. Results of numerical simulation of the supercritical fluid outflow on thermodynamic equilibrium and non-equilibrium relaxation models of phase transition with different relaxation times have been compared. It is shown that the application of the proposed model at this stage leads to qualitatively correct results. This allows us specifying the ways for further development and improvement of the model.

The work was financially supported by the Russian Science Foundation (grant No. 14-29-00093).

\section{References}

1. M.V. Alekseev, I.S. Vozhakov, S.I. Lezhnin, N.A. Pribaturin, Thermophys. Aeromech. 23, 869 (2016)

2. I.S. Vozhakov, M.V. Alekseev, S.I. Lezhnin, N.A. Pribaturin, J. Physics: Conference Series 754032021 (2016)

3. V.I. Artemov, K.B. Minko, G.G. Yankov, Thermal Eng. 62897 (2015)

4. R.H. Bolotnova, V.A. Buzina, Computational Continuum Mechanics 7, 14 (2014) [in Russain]

5. P. Zapolski, Z. Bilicky, L. Bolle, J. Franco, Int. J. Multiphase Flow 22, 473 (1996)

6. S.I. Lezhnin, I.S. Vozhakov, M.V. Alekseev, A.R. Bogomolov, Vestnik of Kuzbass State Technical University 5 (2016)

7. R.I. Nigmatulin, Dynamics of multiphase media (1987) [in Russain]

8. V.G. Levich, Physico-chemical hydrodynamics (1959) [in Russain]

9. L.D. Landau, E.M. Lifshits, Hydrodynamic (1986) [in Russain]

10. G.A. Saltanov, Supersonic two phase flows (1972) [in Russain] 